\title{
Fusion of Visible and Infrared Image Using Adaptive Tetrolet Transform
}

\author{
Kaifeng Liu ${ }^{1, a}$, Baohong Yuan ${ }^{2, b}$, Dexiang Zhang ${ }^{1, c}$ and Jingjing Zhang ${ }^{1, d}$ \\ ${ }^{1}$ The School of Electrical Engineering and Automation of Anhui University, Hefei, 230601, China \\ ${ }^{2}$ The School of Electronic and Electrical Engineering of Anhui Sanlian University, Hefei, 230601, \\ China \\ a kfliu@ahu.edu.cn, ${ }^{b}$ baohong_yuan@163.com, ${ }^{c}$ zdxdzxy@126.com, ${ }^{d}$ helenzjj@aiofm.ac.cn
}

\begin{abstract}
Keywords: Visible and infrared image, tetrolet transform, image fusion, edge information measurement factor
\end{abstract}

\begin{abstract}
A new region-based image fusion technique using the adaptive tetrolet transform is proposed to produce a fused image better for human and machine interpretation. Firstly, the visible image and infrared image can be decomposed into low-frequency and high-frequency coefficients using tetrolet transform. For the low-frequency coefficients, the average fusion method is used. For the each directional high frequency sub-band coefficients, the larger region edge information measurement factor is used to select the better coefficients for fusion. At last the fused image can be obtained by utilizing inverse transform for fused tetrolet coefficients. Experimental results show that the proposed algorithm gives more satisfactory results than the traditional image fusion algorithms in preserving the edges and texture information.
\end{abstract}

\section{Introduction}

Infrared images can often provide information missing in visible images in a night vision system, but an infrared image does not provide all of the information necessary at night. Features such as road color are completely missing from infrared images and features such as object target marking infrared may be hard to identify or are completely missing in infrared image. Image fusion techniques can be used to combine the relevant information from both the visible and infrared images ${ }^{[1]}$.

The aims of data fusion are the reduction of uncertainty and increase in accuracy in a decision task. This is accomplished by incorporation of several sources of information. The current definition of sensor fusion is very broad and the fusion can take place at the signal, pixel, feature, and symbol level. Image fusion on the pixel level is the combination of two or more different images to form a new image. Pixel level image fusion can be helpful for a human observer to more easily detect and recognize potential targets ${ }^{[2]}$.

Over the last decade, wavelet transform has been widely applied in the field of image fusion and provided good processing affect. However, the wavelet transform fails to achieve optimal results with images that contain geometric structures in other directions ${ }^{[3]}$. Thus the wavelet transform is not the optimal choice for describing images.

In the last years a lot of methods have been proposed to improve the treatment of orientated geometric image structures. Curvelets, contourlets, shearlets, and directionlets are wavelet systems with more directional sensitivity ${ }^{[4]}$.

This paper introduces a new image fusion method based on tetrolet transform. We introduce a new adaptive algorithm whose underlying idea is simple but very fast and effective. The proposed method is especially designed for sparse image approximation due to the non-redundance of the basis functions ${ }^{[5]}$. A combination of the infrared with a classical visible image of the same scene can be used to detect the target and precisely identify its location. 


\section{Tetrolet transform}

Tetrolets are Haar-type wavelets whose supports are tetrominoes which are shapes made by connecting four equal-sized squares. We divide the image into $4 \times 4$ blocks, and then we determine in each block a tetromino partition which is adapted to the image geometry in this block. Tetrominoes are shapes made by connecting four equal-sized squares, each joined together with at least one other square along an edge ${ }^{[6]}$.

We start with the input image $a^{0}=(a[i, j])_{i, j=0}^{N-1}$ with $N=2^{J}, J \in N$. In the r th-level, $r=1,2, \ldots, J-1$, we apply the following computations.

1. Divide the low-pass image $\mathrm{a}^{r-1}$ into blocks $\mathrm{Q}_{i, j}$ of $i, j=0,1, \ldots, N / 2^{r+1}-1$.

2. Find in each block the sparsest tetrolet representation. In each block $\mathrm{Q}_{i, j}$ we compute the pixel averages for every admissible tetromino covering $c=1,2, \ldots, 117$, we can determine the lowpass part

$$
\mathrm{a}^{\mathrm{r},(\mathrm{c})}[s]=\sum_{(m, n) \in I_{s}^{(c)}} \varepsilon[0, L(m, n)] a^{r-1}[m, n]
$$

as well as the three high-pass parts for $l=1,2,3$

$$
w_{l}^{r,(c)}=\left(\sum_{(m, n) \in I_{s}^{(c)}} \varepsilon[l, L(m, n)] a^{r-1}[m, n]\right)_{s=0}^{3}
$$

where the coefficients $\varepsilon[l, m], l, m=0,1,2,3$ are entries from the Haar wavelet transform matrix

$$
W=(\varepsilon[l, m])_{l, m=0}^{3}=\frac{1}{2}\left[\begin{array}{cccc}
1 & 1 & 1 & 1 \\
1 & 1 & -1 & -1 \\
1 & -1 & 1 & -1 \\
1 & -1 & -1 & 1
\end{array}\right]
$$

Then we choose the covering $c^{*}$ such that the $l_{1}$-norm of the tetrolet coefficients becomes minimal

$$
c^{*}=\arg \min _{c} \sum_{l=1}^{3} \sum_{s=0}^{3}\left|w_{l}^{r,(c)}[s]\right|
$$

Hence, for every block $\mathrm{Q}_{i, j}$ we get optimal tetrolet decomposition $\left[a^{r,\left(c^{*}\right)}, w_{1}^{r,\left(c^{*}\right)}, w_{2}^{r,\left(c^{*}\right)}, w_{3}^{r,\left(c^{*}\right)}\right]$.

3. In order to be able to apply further levels of the tetrolet decomposition algorithm, rearrange the low-pass and high-pass coefficients of each block into a $2 \times 2$ block.

$$
a_{\mid Q_{i, j}}^{r}=R\left(a^{r,\left(c^{*}\right)}\right)=\left[\begin{array}{ll}
a^{r,\left(c^{*}\right)}[0] & a^{r,\left(c^{*}\right)}[2] \\
a^{r,\left(c^{*}\right)}[1] & a^{r,\left(c^{*}\right)}[3]
\end{array}\right]
$$

and in the same way $w_{l \mid Q_{i, j}}^{r}=R\left(w_{l}^{r,\left(c^{*}\right)}\right)$

4. After finding a sparse representation in every block $\mathrm{Q}_{i, j}$, we store the low-pass matrix $\mathrm{a}^{r}$ and the high-pass matrices $w_{l}^{r}$, replacing the low-pass image $a^{r-1}$ by the matrix

$$
a^{r-1}=\left[\begin{array}{ll}
a^{r} & w_{2}^{r} \\
w_{1}^{r} & w_{3}^{r}
\end{array}\right]
$$

5. Apply step 1 to 4 to the low-pass image we can get multi-scale and multi-direction tetrolet decompose.

\section{Tetrolet Based the Visible Image and Infrared Image Fusion}

The goal of using a transform based method of combining source images is to obtain an image better than that produced by simply averaging the source images or an image obtained by taking the 
maximum pixel value at each location in the source images. We apply the tetrolet transform to image fusion so as to create new fused images that have more information than the source images.

Tetrolet decomposition of the each original image separates the image into low frequency regions and high frequency regions. The low frequency sub-band image coefficients shows the image approximate characteristic ${ }^{[7]}$. The high frequency regions correspond to edges in the source image. For the approximate coefficients fusing, the fused approximate coefficients are obtained with average method. The fusion rule can describe as:

$$
T_{F}(m, n)=\left(T_{A}(m, n)+T_{B}(m, n)\right) / 2
$$

where $T_{A}(m, n)$ and $T_{B}(m, n)$ is low coefficients of image $A$ and image $B$ respectively. $T_{F}(m, n)$ is low coefficients of fused image.

In tetrolet transform, divide the low-pass image into blocks of size $4 \times 4$, we obtain four low-pass coefficients and twelve tetrolet coefficients using harr wavelet transform. The tetrolet transform selects the optimal covering in each image block from the alphabet $\{1, \ldots, 117\}$. For the coefficients of the high frequency, larger absolute values of multi-scale decomposition coefficients correspond to sharper brightness changes such as edges, lines and region boundaries. For the coefficients of the highest frequency, fusions by the rule of choosing the greater of the edge information measurement with the region consistency check ${ }^{[8]}$.

The response of the ratio edge detector between two regions $i$ and $j$ of radiometric means $\mu_{i}$ and $\mu_{j}$ is defined as $\gamma_{i j}$ :

$$
\gamma_{i j}=1-\min \left(\frac{\mu_{i}}{\mu_{j}}, \frac{\mu_{j}}{\mu_{i}}\right)
$$

The edge information measurement factor is $\gamma=\min \left(\gamma_{12}, \gamma_{23}\right)$, the minimum response of a ratio edge detector on both sides (with indexes 1 and 3 ) of the linear structure (with index 2).

Therefore fusion rule is to select the larger absolute value of the two coefficients at each pixel. First we calculate the $12 \times 1$ tetrolet high frequency coefficients energy. Then the coefficient with larger edge information measurement factor is used in the following fusion process, while the coefficient with less local energy is discarded:

$$
\begin{gathered}
T_{F}(i, j)= \begin{cases}T_{A}(i, j) & \gamma_{A}(i, j) \geq \gamma_{B}(i, j) \\
T_{B}(i, j) & \gamma_{B}(i, j) \geq \gamma_{A}(i, j)\end{cases} \\
C_{F}(i, j)= \begin{cases}C_{A}(i, j) & \gamma_{A}(i, j) \geq \gamma_{B}(i, j) \\
C_{B}(i, j) & \gamma_{B}(i, j) \geq \gamma_{A}(i, j)\end{cases}
\end{gathered}
$$

where $(i, j)$ and $\gamma(i, j)$ denotes the current position of tetrolet coefficient edge information measurement factor respectively. $C(i, j)$ denotes the current position of tetrolet coefficient covering number in each image block.

\section{Experiments and Results}

In order to test our image fusion algorithm, we apply a complete tetrolet decomposition of the visible image and infrared image and use the methods proposed for image fusion. Figure (1) shows the original the visible image and the infrared source image as well as their fused image using the two image fusion algorithms. 


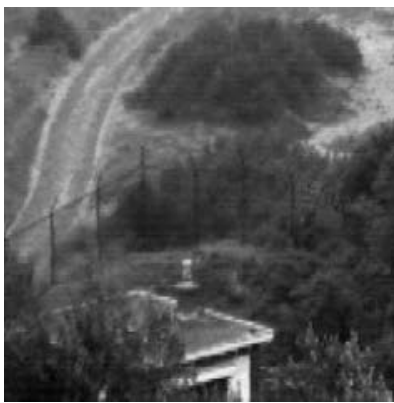

(a) The visible image

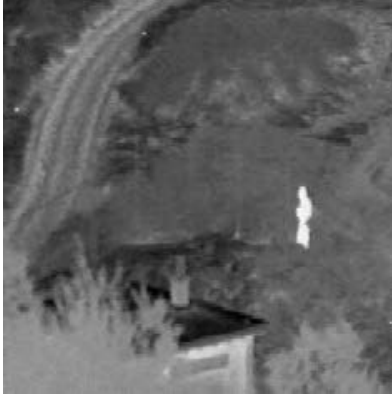

(b) the infrared image

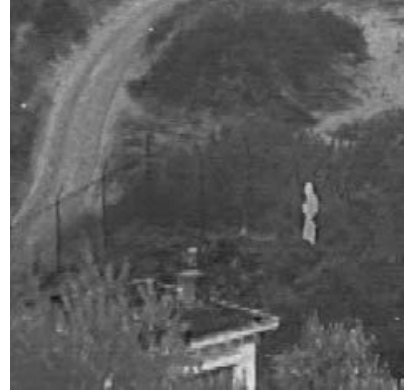

(c) fused image by WT

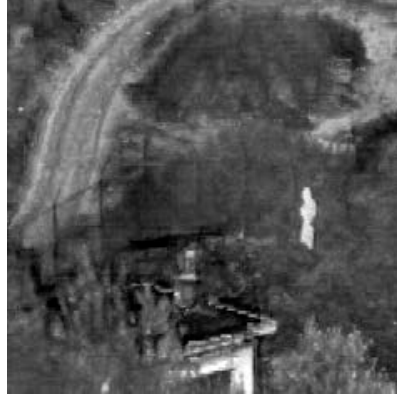

(d) proposed method

Figure 1: Fused image with the different methods. (a) The visible image, (b) the infrared image, (c) fused image by WT, (d) fused image by proposed method

In order to reveal the effects of different algorithms, the different fusion methods are compared with that of the Wavelet transforms method. Criteria values of fused images by different methods are shown in Table 1

Table 1 Criteria value of fused images by different methods

\begin{tabular}{c|c|c|r|r|r}
\hline \multicolumn{1}{c}{$\begin{array}{c}\text { Criteria } \\
\text { method }\end{array}$} & \multicolumn{1}{c}{$\begin{array}{c}\text { average } \\
\text { value }\end{array}$} & $\begin{array}{c}\text { standard } \\
\text { deviation }\end{array}$ & \multicolumn{2}{c}{$\begin{array}{c}\text { the mean } \\
\text { entropy } \\
\text { cross entropy }\end{array}$} & $\begin{array}{c}\text { the root } \\
\text { cross entropy }\end{array}$ \\
\hline WT & 103.582 & 28.532 & 6.682 & 0.818 & 0.970 \\
TT & 83.604 & 34.656 & 6.980 & 0.271 & 0.308 \\
\hline
\end{tabular}

\section{Conclusion}

We have presented an improved method of image fusion based on tetrolet transform. Tetrolet transform represents edges better than wavelet and it is well suited for the visible image and infrared image fusion. Experimental results show that our algorithm is a good try on image fusion. The results we obtained are very similar to these obtained with the traditional algorithm. We designed edge information measurement factor fit to the tetrolet multiscale representation. Experiments and their studies were conducted by applying the proposed method, and also other image fusion methods. Compared with the fusion method based on wavelet and other methods, the experiment shows that this approach can achieve better result than them.

\section{Acknowledgment}

This work was financially supported by the Chinese National Science Foundation Grant (No.61272025 \& No.61402004) and the Nature Science Foundation of Anhui Province Education Department under Grant (No. KJ2012B065) and the Nature Science Foundation of Anhui Sanlian University Grant (No. 13ZLGC043 \& 2014Z020) and the revitalization plan project of higher education of Anhui Province Grant (No. 2013zytz082).

\section{References}

[1] Wald, L., Ranchin, T. and Mangolini, M., Photogrammetric Engineering \& Remote Sensing. Vol. 63(1997), p. 691

[2] Myungjin Choi, Rae Young Kim,etal. IEEE Geoscience and Remote Sensing Letters, Vol.2 (2005), p. 136.

[3] Piella G. Information fusion, Vol. 4 (2003), p. 259.

[4] M. N. Do and M. Vetterli, IEEE Trans. on Image Processing, Vol.14 (2005), p. 2091.

[5] Zhang Jingjing, Fang Yonghua. Opto-Electronic Engineering, Vol. 34 (2007), p.78. 
[6] S.W. Golomb. Polyominoes. Princeton University Press, 1994..

[7] Liu Gang, Jing Zhongliang, Sun Shaoyuan., Journal of Zhejiang University Science, Vol. 7 (2006), p. 117.

[8] Naidu, V. P. S., Raol, J. R., Defence Science Journal, vol. 58 (2008), p. 338. 